\title{
A Performance Study of Limited Range Partial Wavelength Conversion for Asynchronous Optical Packet/Burst Switching
}

\author{
Kaan Dogan and Nail Akar \\ Electrical and Electronics Engineering Department \\ Bilkent University, Ankara, Turkey \\ Email: \{kaan,akar\}@ee.bilkent.edu.tr
}

\begin{abstract}
In this work, we study an asynchronous optical packet/burst switching node equipped with a number of limited range wavelength converters shared per output link. A wavelength conversion policy is one by which the outgoing wavelength for an optical packet is selected if its incoming wavelength is in use. Through simulations, we show that the so-called "far conversion" policy in which the optical packet is switched onto the farthest available wavelength in the tuning range, outperforms the other policies we studied. We point out the "clustering effect" in the use of wavelengths to explain this phenomenon.
\end{abstract}

\section{INTRODUCTION}

Optical data transmission over fiber lines with the Wavelength Division Multiplexing (WDM) technology helps network operators to cope with rapidly increasing traffic demands. WDM allows multiplexing of several wavelength channels on a single fiber and WDM systems with 32 or 64 channels each operating at $10 \mathrm{Gbps}$ for a total capacity of 320 or 640 Gbps over a single fiber are common today. Two new packet-based optical switching paradigms have recently been introduced to make efficient use of bandwidth: Optical Packet Switching (OPS) [1], [2] and Optical Burst Switching (OBS) [3], [4]. OPS requires line-rate header parsing and is viewed as a longer term solution due to the current technological limitations in packet header processing [2]. OBS, on the other hand, eliminates the need for header parsing by segregating the control and data planes. In OBS, the reservation request for a burst is signalled out of band by the use of a burst control packet and processed in the electronic domain whereas the burst itself is transported end-to-end all in the optical domain. Since we're interested only in the data plane, we will use the common term "(optical) packet" and "(optical) packet switching" to refer to a packet/burst and the data planes of OPS/OBS, respectively.

In synchronous (i.e., time-slotted) optical packet switched networks, packet lengths are fixed and packets are assumed to arrive at slot boundaries. Such models are relatively difficult to implement due to the need for expensive synchronization equipment. In asynchronous (i.e., unslotted) networks, optical packet lengths are variable and packets arrive asynchronously and therefore there is not a need for costly synchronization equipment. On the down side, performance evaluation of asynchronous packet switching networks require advanced probabilistic methods and are generally more difficult. Our focus in this study is on asynchronous optical packet switching.

One of the major issues in optical packet switching networks is contention which arises as a result of two or more incoming packets contending for the same output wavelength. Contention is resolved either in wavelength domain by wavelength converters, in time domain by Fiber Delay Lines (FDL), or in space domain by deflection routing [3]. If contention cannot be resolved by any one of the proposed techniques, then one or more contending packets would be blocked. In this paper, we concentrate our attention to only Tunable Wavelength Converters (TWC) that are used to switch optical packets from one wavelength to another for contention resolution. In Full Wavelength Conversion (FWC), a packet arriving at a certain wavelength can be switched onto any other wavelength towards its destination. FWC reduces packet blocking probabilities significantly when compared with the case of No Wavelength Conversion (NWC) [5]. In Partial Wavelength Conversion (PWC), there is a limited number of TWCs, and consequently some optical packets cannot be switched towards their destination and therefore blocked when all converters are busy despite the availability of free wavelength channels on the output link. In PWC, TWCs may be collected as a single converter pool for converter sharing across all fiber lines, which is referred to as the Share-Per-Node (SPN) architecture [6]. A simpler architecture allows separate TWC banks per output fiber line and the corresponding solution is called the Share-Per-Line (SPL) architecture [6] which is depicted in Fig. 1. The cost-effective SPL solution has its advantages of low-complexity switching matrix, significant performance and TWC saving gain compared to other alternatives, in addition to its amenability to exact stochastic analysis [7].

Another separate issue in wavelength conversion is whether there is a specified range of wavelengths that a given wavelength can be converted to. Full Range TWCs (FR-TWC) do not have any tuning range limit and they can convert an incoming wavelength to any other wavelength. In limited-range wavelength conversion, a burst arriving on a wavelength can be converted to a fixed set of wavelengths above and below the original wavelength and such TWCs are called Limited-Range TWC (LR-TWC) [8]. The degree of conversion $d$ is defined 


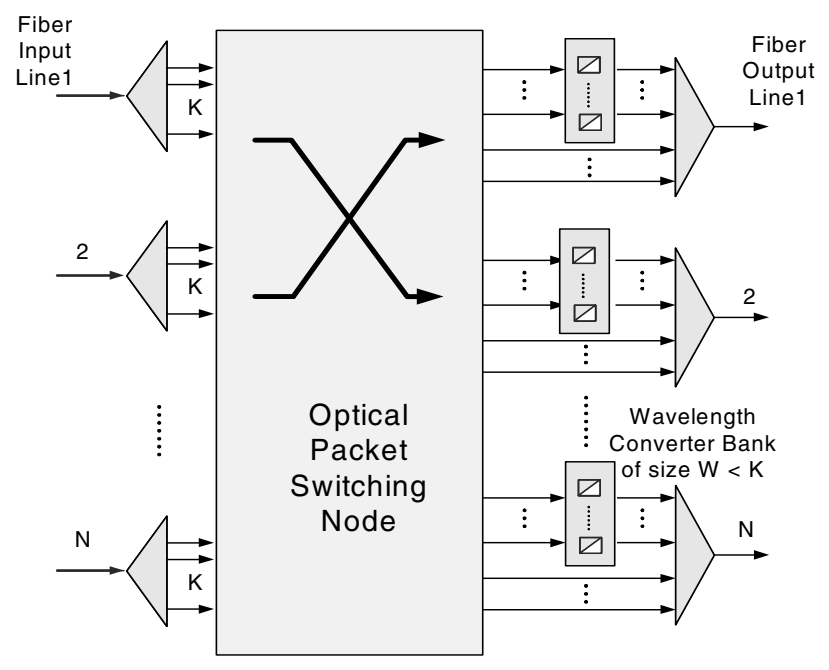

Fig. 1. The general architecture of an optical packet switching node with $N$ fiber I/O lines, $K$ wavelength channels on each fiber line, and a bank of wavelength converters of size $W$ shared per output link

as the total number of wavelengths available on both sides of the original wavelength and therefore an incoming optical packet can either be converted to one of the $d$ destination wavelengths in the physical neighbourhood or can stay on the same wavelength if the latter is available. In particular, we will study the circular-type limited-range wavelength scheme depicted in Fig. 2 for which the Conversion Range (CR) of an incoming wavelength $i, 0 \leq i \leq K-1$, denoted by $C R(i)$ is given as the following wavelength set:

$$
\begin{aligned}
C R(i)= & \left\{\bmod \left(i-\frac{d}{2}, K\right), \ldots, \bmod (i-1, K),\right. \\
& \left.\bmod (i+1, K), \ldots, \bmod \left(i+\frac{d}{2}, K\right)\right\} .
\end{aligned}
$$

In the above definition, $\bmod (a, b)$ is the remainder on division of $a$ by $b$ for given integers $a$ and $b$. Revisiting Fig. 2 for the case of $d=4$, the incoming boundary wavelength 0 can be converted to the set $\{K-2, K-1,1,2\}$ and the incoming wavelength $i$ can be converted to the set $\{i-2, i-1, i+1, i+2\}$ assuming $2 \leq i \leq K-2$.

Circular-type limited-range conversion preserves the symmetry among wavelengths and is therefore the preferred conversion scheme of this paper although we believe the results of the paper apply to more general and realistic limited-range wavelength conversion schemes.

In this paper, we will study the performance of an asynchronous optical packet switch employing PWC on a shareper-link basis with the shared TWCs being of LR type; see Fig. 1. Due to the way converters are shared, each outgoing link can be studied independently. We assume that $N$ is large enough so that the packet traffic destined to a specified outgoing link can be modelled with the Poisson process with rate $\lambda$. The number of channels on the given link is denoted by $K$ and a converter bank of size $W \leq K$ is assumed to be available for that link. As a usual assumption, the wavelength distribution of the incoming request is assumed

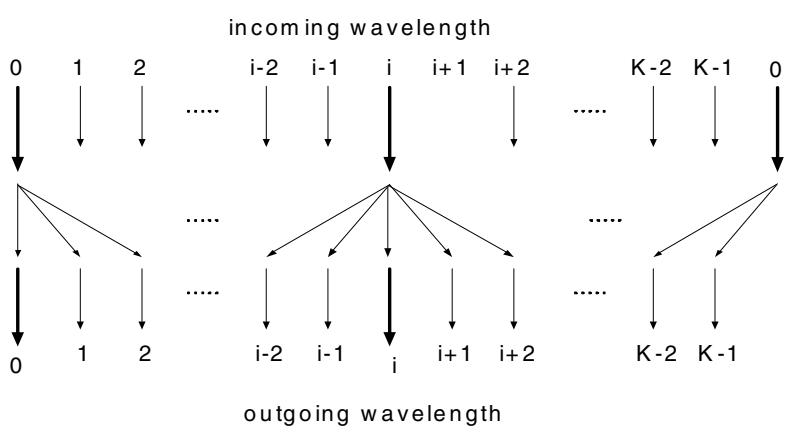

Fig. 2. The circular conversion scheme depicted for $d=4$ for the boundary incoming wavelength 0 and a wavelength $i$ far from the boundaries

to be uniform among all the wavelengths. We also adhere to the exponential packet length distributions which is common in the literature where the mean packet length is $1 / \mu$. An exact numerical analysis procedure is proposed for the same problem for the case of FR-TWCs in [7]. However, a similar analysis does not seem to be plausible for the case of LRTWCs due to the way conversion ranges overlap. Moreover, random conversion to any of the available wavelengths in the case of FR-TWCs provides the best results due to the uniform distribution of the incoming wavelengths. However, conversion policies substantially impact the performance for the case of LR-TWCs. The goal of this paper is to study different simple-to-implement wavelength conversion policies for the case of LR-TWCs. On the other hand, we give an approximate analytical procedure based on [7] to give a lower bound on the packet blocking probability with an auxiliary model that captures part of the complex system dynamics.

The outline of the paper is as follows. We introduce a number of simple-to-implement wavelength conversion policies in Section II. We provide an approximate analysis tool so as to provide a lower bound on the blocking probabilities for the SPL LR-TWC architecture in Section III. The simulation- and analysis-based results are given in Section IV. We conclude in the final section.

\section{Conversion Policies for Limited-RAnge TWCS}

The analysis carried out in [7] involves FR-TWCs in which a wavelength is selected randomly out of the set of idle wavelengths in case the incoming wavelength is occupied. This randomized policy is best for FR-TWCs when the wavelength of the incoming packets is uniformly distributed. However, such a randomized policy has drawbacks in the case of LR-TWCs even for the case of uniformly distributed wavelengths. We explain this phenomenon by the following scenario. Consider the arrival instance of an optical packet $x$ whose incoming wavelength $i$ is occupied in the outgoing link and therefore the packet requires wavelength conversion. This shows that either a packet had arrived on the same wavelength $i$ and it is still being served or a packet had arrived on a different wavelength $j$ but converted to $i$ since $j$ was occupied. Since conversions take place within a range of the incoming wavelength, the probability of packet $x$ to 
find its conversion range fully occupied is larger than the full occupancy probability of an arbitrarily selected set of $d$ wavelengths other than the wavelength $i$. Equivalently, there is a positive spatial correlation between the status of two neighbouring wavelengths and consequently occupied wavelengths tend to cluster in time as opposed to the case of FR-TWCs. The so-called clustering phenomenon obviously has a detrimental impact on blocking performance. On the other hand, the clustering effect can be reduced by appropriate wavelength conversion policies. We study the following three simple-toimplement wavelength conversion policies at the instance of a packet arrival whose incoming wavelength is occupied.

- Random Conversion: The outgoing wavelength is selected randomly from the set of idle wavelengths in the range.

- Near Conversion: We choose the nearest available wavelength from the set of idle wavelengths in the conversion range and if there exist two such wavelengths, one of them will be selected in random. However, such a policy works in favour of the clustering effect relative to the random conversion policy.

- Far Conversion: In this policy, the farthest available wavelength is selected from the set of idle wavelengths in the conversion range. If there exist two such wavelengths, one of them will be selected in random. Obviously, this policy counteracts the clustering effect.

We explain the clustering effect through a simulation study for which circular wavelength conversion is used for a scenario corresponding to $K=33, W=15, d=8$, and a load of $27 \%$. We assume that the wavelengths are indexed as $0, \ldots, 32$. We concentrate our attention on the middle wavelength numbered 16 and mark the instances for which packets arriving on wavelength 16 find that wavelength in use. We note the occupancy probability of the other wavelengths at these embedded epochs. Our results are depicted in Fig. 3. All conversion policies tend to produce a wavelength occupancy probability histogram clustered within the conversion range of the incoming wavelength. However, such clustering is most dominant in the near conversion policy. We also note that the far conversion policy produces occupancy probability peaks at wavelengths 12 and 20 (i.e., $d / 2$ units away from the incoming wavelength) which are still within the conversion range of wavelength 16 . However, the second and third-order harmonics at wavelengths 8 and 24, and 4 and 28, respectively, generated by the far conversion policy, appear to increase the probability of an arriving packet on wavelength 16 to find at least one idle wavelength in its conversion range.

We note that these policies do not affect the steady-state outgoing wavelength distributions because of the symmetry among the wavelengths. Moreover, all three policies obviously result in identical performance for the particular cases $d=$ 2 and $d=K-1$. In the former case, there are only two adjacent candidate wavelengths for conversion and all three policies collapse to a random conversion policy. The case of $d=K-1$ reduces to full-range conversion case in which we do not observe clustering. The goal of the simulation study

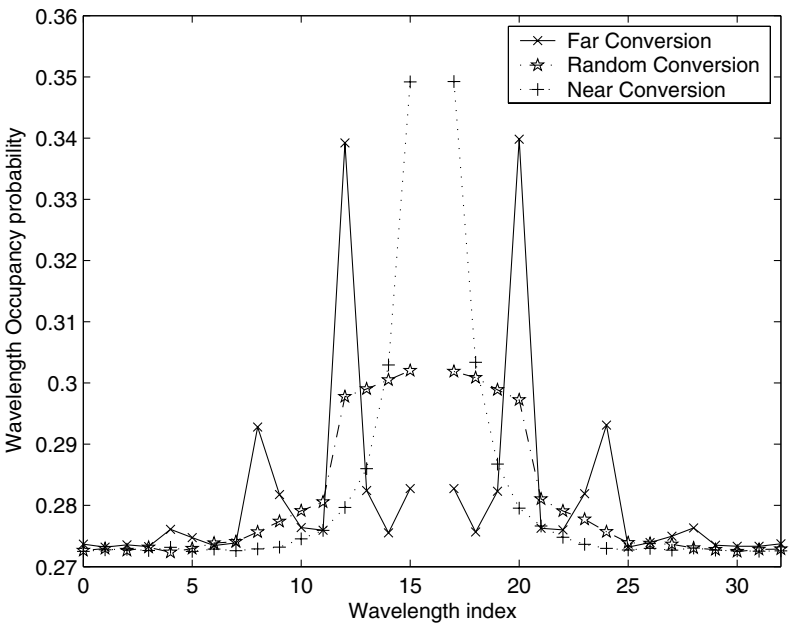

Fig. 3. The occupancy probabilities of wavelengths conditioned upon an arriving packet on wavelength 16 finding this wavelength in use

is to show if there is a notable difference among the three conversion policies for $2<d<K-1$, i.e. moderate degree of conversion.

\section{Analytical Model}

An exact stochastic analysis for limited range wavelength conversion under the three proposed policies does not appear to be plausible and we'll resort to simulations for comparative performance evaluations. However, as a reference we propose the following simple auxiliary model that captures part of the system dynamics. In this model, the conversion range is not the actual $d / 2$ neighbourhood of the incoming wavelength but instead a set of arbitrarily selected $d$ wavelengths at each time conversion takes place. This model captures the impact of degree of conversion but does not accommodate the clustering effect. Since the model is cluster-free we conjecture that it provides a lower bound on the blocking probabilities achievable by limited-range wavelength conversion. Moreover, this model is amenable to exact probabilistic analysis based on the procedure in [7]. For the sake of completeness, we present below the exact analysis method for the auxiliary model. In this model, an incoming optical packet

- is forwarded without conversion if its incoming wavelength is idle on the outgoing link

- is directed to the outgoing link after wavelength conversion if there exist both an available TWC an idle wavelength in the conversion range which, in this auxiliary model, is a randomly selected set of $d$ wavelengths other than the incoming wavelength.

- is blocked otherwise

Under the assumptions, let $i(t)$ and $j(t)$ denote the number of wavelength channels and the number of TWCs that are in use at time $t$, respectively. The process $X(t)=\{(i(t), j(t))$ : $t \geq 0\}$ is a Continuous Time Markov process on the state space $S=\{(i, j): 0 \leq i \leq K, 0 \leq j \leq \min (i, W)\}$. To show this, let us assume that the process is in some state $(i, j), 0 \leq$ 
$i<K, 0 \leq j \leq \min (i, W)$ at time $t$. If a new burst arrives in the interval $(t, t+\delta)$ which occurs with probability $\lambda \delta+o(\delta)$ (i.e., $\lim _{\delta \rightarrow 0} o(\delta) / \delta=0$ ) [9], there are three possibilities:

A1) the wavelength on which the burst is riding on is not currently used on the link which occurs with probability $(K-i) / K$ and the burst will be admitted and the process will jump to $(i+1, j)$ at time $t+\delta$,

A2) that wavelength is already used with occurs with probability $i / K$

- then if $j=W$ then the burst will be blocked because the converter pool is all busy leading to no state change,

- else if $j<W$ and all of the wavelength channels that are in the conversion range of the riding wavelength are used which occurs with probability

$$
\frac{\left(\begin{array}{c}
K-d-1 \\
i-1-d
\end{array}\right)}{\left(\begin{array}{c}
K-1 \\
i-1
\end{array}\right)} \text { if } i \geq d+1,
$$

then the burst will be blocked.

- else the conversion range has at least one free wavelength available then the packet will be admitted on one of the available wavelengths randomly using one of the free converters and the process will make a transition to state $(i+1, j+1)$ at time $t+\delta$.

Assume now that the process $X(t)$ is currently in some state $(i, j), 0<i \leq K, 0 \leq j \leq \min (i, W)$ at time $t$. If a burst departs in the interval $(t, t+\delta)$ which occurs with probability $i \mu \delta+o(\delta)$ then there are two possibilities:

B1) a TWC was used for this burst which occurs with probability $j / i$ and the process $X(t)$ will jump to state $(i-1, j-1)$ at at time $t+\delta$,

B2) a TWC was not used at all for this departing burst which occurs with probability $(i-j) / i$ and the process $X(t)$ will make a transition to state $(i-1, j)$ at time $t+\delta$.

When the process $X(t)$ is in state $(0,0)$, then there cannot be any departures. It is thus clear that the process $X(t)$ is a Continuous Time Markov Chain (CTMC) and the infinitesimal generator of the CTMC possesses a block-tridiagonal form if the states are properly enumerated as in [7]. A numerically stable and efficient solution procedure, the so-called block tridiagonal LU factorization algorithm [10] can then be used to find the stationary solution of the underlying CTMC while taking advantage of the block-tridiagonal structure of the generator.

For obtaining the packet blocking probabilities, we observe that a new packet arrival is blocked if

- the Markov chain resides in $(K, j), 0 \leq j \leq W$ (i.e. all wavelength channels are in use)

- the Markov chain resides in state $(i, W), W \leq i<K$ (i.e. all converters are in use) and the incoming wavelength is occupied (this occurs with probability $i / K$ )

- the Markov chain resides in states $(i, j), d<i<K, 0 \leq$ $j<W$, the incoming wavelength is occupied and the conversion range is fully occupied which occurs with probability

$$
\frac{i}{K} \frac{\left(\begin{array}{c}
K-d-1 \\
i-1-d
\end{array}\right)}{\left(\begin{array}{c}
K-1 \\
i-1
\end{array}\right)}
$$

By using the PASTA (Poisson Arrivals See Time Averages) property [9], the packet blocking probability $P_{b}$ of the auxiliary model can be written as

$$
\begin{aligned}
P_{b} & =\sum_{j=0}^{W} x_{K, j}+\sum_{i=W}^{K-1} \frac{i}{K} x_{i, W} \\
& +\sum_{i=d+1}^{K-1} \sum_{j=0}^{W-1} x_{i, j} \frac{i}{K} \frac{\left(\begin{array}{c}
K-d-1 \\
i-1-d
\end{array}\right)}{\left(\begin{array}{c}
K-1 \\
i-1
\end{array}\right)}
\end{aligned}
$$

where $x_{i}=\left(x_{i, 0}, x_{i, 1}, \cdots, x_{i, W}\right)$ for $i \geq W, x_{i, j}$ is the steady-state probability of having $i$ wavelengths and $j$ TWCs occupied.

\section{NumERICAL RESUlTS}

In this study, we obtain the packet blocking probabilities for the three wavelength conversion policies by simulations. We also solve for the auxiliary model analytically using the technique described in the previous section. The goal of this simulation study is to comparatively study the three policies and compare them against the auxiliary model which is clustering-free. Without loss of generality, the mean burst time $1 / \mu$ is normalized to unity in all numerical examples presented below. Each simulation result is obtained by averaging 10 independent runs. We do not present blocking probabilities less then $10^{-9}$. We define the system load $\rho=\lambda / \mu K$, wavelength conversion ratio $r=100 \frac{W}{K}$ and tuning range ratio $\gamma=100 \frac{d}{K}$.

In Fig. 4, we fix the number of channels $K$ to 16 and we illustrate the packet blocking probabilities with respect to the wavelength conversion ratio $r$ for different values of the tuning range ratio $d$ and for two values of the load $\rho$; the upper set of curves is for $\rho=0.5$ and the lower set for $\rho=0.25$ for all the figures presented in this paper. Independent of the conversion policy used, we observe improved utilization of the TWCs with increasing tuning range. We also observe that for small tuning range ratios, it is not as necessary to use large wavelength conversion ratios as in the case of large tuning range ratios. As expected, the three policies provide similar results for both small and large values of $d$ whereas for moderate values of $d$ the "far conversion" policy outperforms the "random conversion" policy which again outperforms the "near conversion" policy. This out-performance is apparent especially for large conversion ratios where the packet losses are mostly due to range occupancy and not the lack of converters. The analytical model, on the other hand, provides a good approximation only when the tuning range ratio is large but it provides a reference lower bound for all the cases studied.

In the second example, we fix $K=32$ and investigate the performance of the three conversion policies for 4 different pairs of $W$ and $\rho$ values in Fig. 5. We observe that the impact of the "far conversion" policy is stronger with increased 


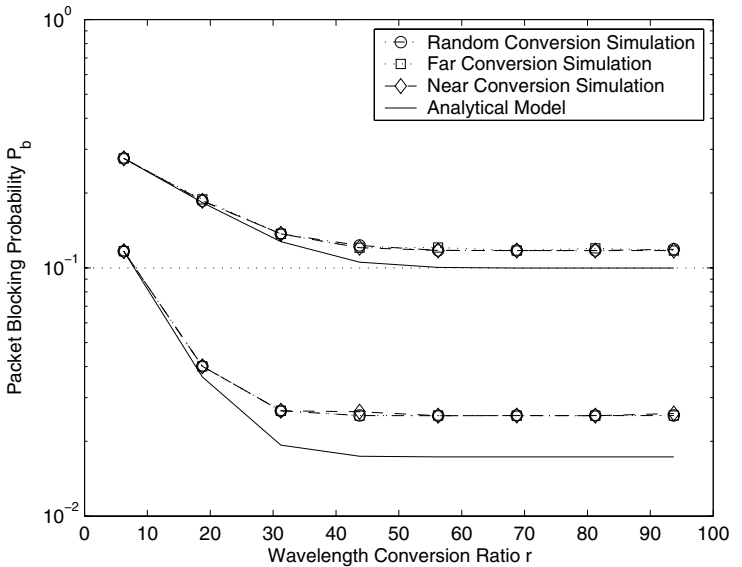

(a)

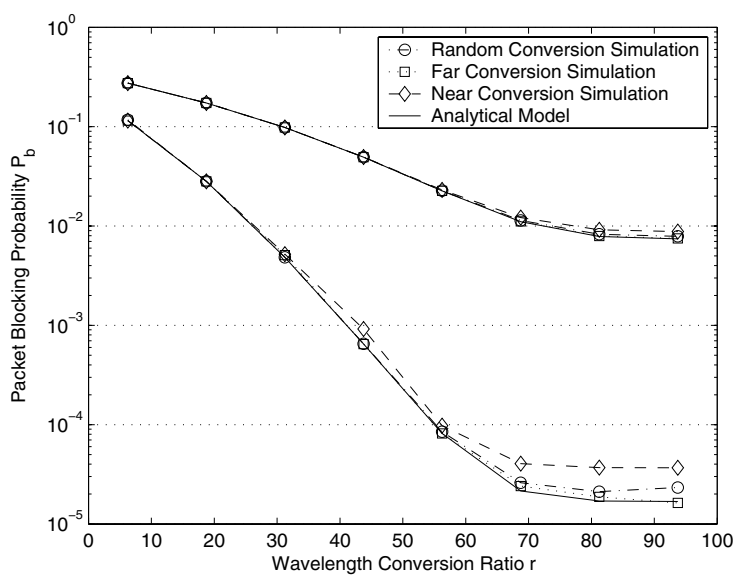

(c)

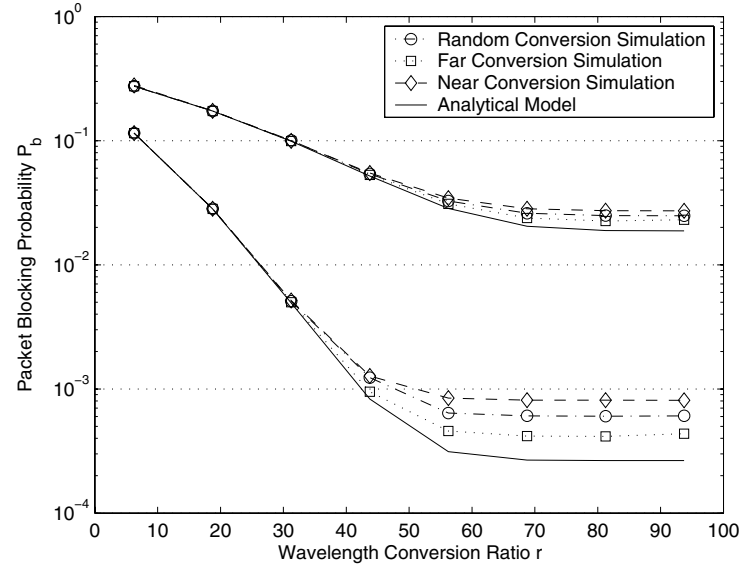

(b)

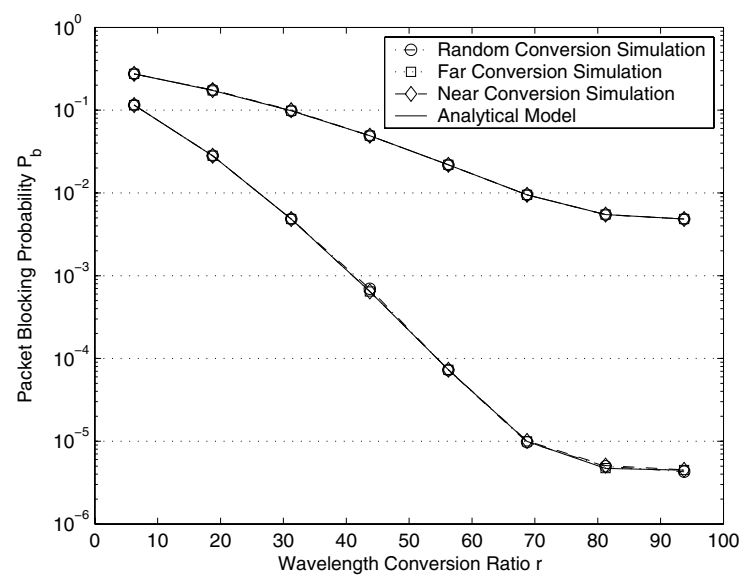

(d)

Fig. 4. Blocking probabilities for $K=16$ with respect to $r$ for two different values of $\rho=0.25$ and $\rho=0.5$ when (a) $d=2$ (b) $d=6$ (c) $d=10$ and (d) $d=14$

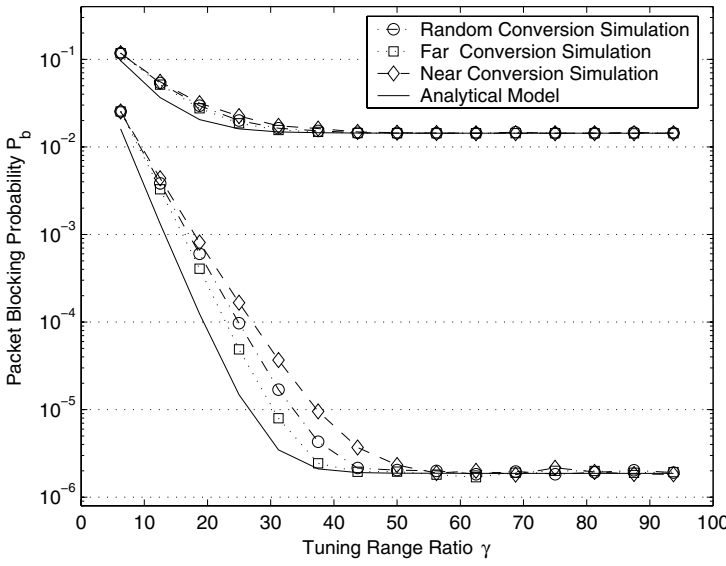

(a)

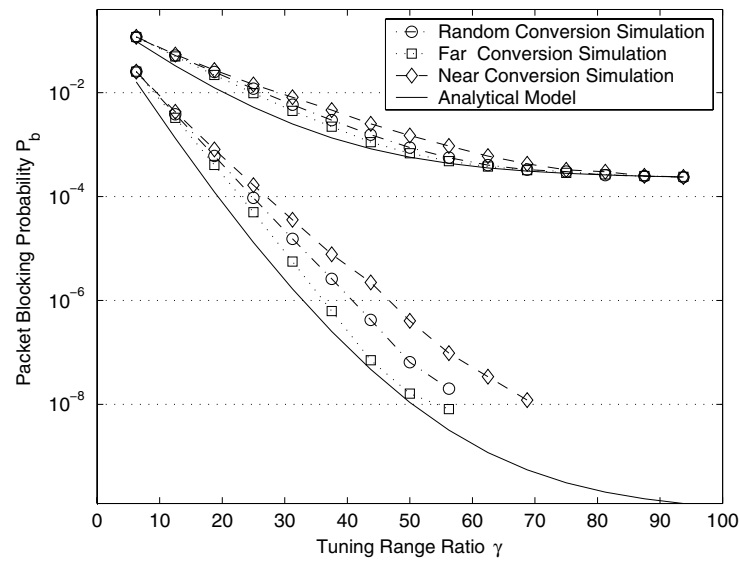

(b)

Fig. 5. Blocking probabilities for $K=32$ with respect to $\gamma$ for two different values of $\rho=0.25$ and $\rho=0.5$ when (a) $W=15$ and (b) $W=25$

wavelength conversion ratios, relatively small tuning range ratios, and at lighter loads.

\section{COnCLusions}

In this paper, we study the performance of asynchronous optical packet switched architectures equipped with a number of limited-range tunable wavelength converters shared on a 
per-output-link basis. We identify the wavelength clustering effect to describe the spatial correlation among the occupied wavelengths which is detrimental to blocking performance in systems with limited-range converters. We propose a "far conversion" policy to reduce the clustering effect and we show through simulations that this proposed policy notably outperforms the other "random" and "near" conversion policies especially in the low load and moderate tuning range ratio regimes. We also introduce a Markovian auxiliary model that captures the effect of the tuning range but not the clustering phenomenon, in order to provide a lower bound on the blocking probabilities for the three studied wavelength conversion policies.

\section{ACKNOWLEDGMENT}

This work is supported in part by The Science and Research Council of Turkey (Tübitak) under project No. EEEAG101E048 and by the Commission of the European Community IST-FP6 e-Photon/ONe Network of Excellence project.

\section{REFERENCES}

[1] P. Gambini, M. Renaud, C. Guillemot, F. Callegati, I. Andonovic, B. Bostica, D. Chiaroni, G. Corazza, S. L. Danielsen, P. Gravey, P. B. Hansen, M. Henry, C. Janz, A. K. R. Krahenbuhl, C. Raffaelli, M. Schilling, and A. T. L. Zucchelli, "Transparent optical packet switching: Network architecture and demonstrators in the EOPS project," JSAC, vol. 16, no. 7, pp. 1245-1259, 1998.
[2] G. N. Rouskas and L. Xu, "Optical packet switching," in Emerging Optical Network Technologies: Architectures, Protocols, and Performance, K. Sivalingam and S. Subramaniam, Eds. Norwell, Massachusetts: Springer, 2004, pp. 111-127.

[3] C. Qiao and M. Yoo, "Optical burst switching (OBS) - a new paradigm for an Optical Internet," Jour. High Speed Networks (JHSN), vol. 8, no. 1, pp. 69-84, 1999.

[4] Y. Chen, C. Qiao, and X. Yu, "Optical burst switching: A new area in optical networking research," IEEE Network Mag., vol. 18, no. 3, pp. 16-23, 2004.

[5] R. A. Barry and P. Humblet, "Models of blocking probability in alloptical networks with and without wavelength changers," IEEE J. Sel. Areas Commun., vol. 14, pp. 858-867, 1996.

[6] V. Eramo, M. Listanti, and P. Pacifici, "A comparison study on the number of wavelength converters needed in synchronous and asynchronous all-optical switching architectures," IEEE Jour. Lightwave Tech., vol. 21, no.2, pp. 340-355, 2003.

[7] N. Akar and E. Karasan, "Exact calculation of blocking probabilities for bufferless optical burst switched links with partial wavelength conversion," in 1st Conference on Broadband Networks (BROADNETS'04), Optical Networking Symposium, 2004, pp. 110-117.

[8] V. Puttasubbappa and H. Perros, "An approximate queueing model for limited-range wavelength conversion in an OBS switch," in Networking 2005, 2005.

[9] L. Kleinrock, Queuing Systems, Vol. 1, Theory. John Wiley, New York, 1989.

[10] G. H. Golub and C. F. van Loan, Matrix Computations, 3rd ed. The Johns Hopkins University Press, 1996. 